\title{
THE CENTER OF THE QUOTIENT DIVISION RING OF THE UNIVERSAL ENVELOPE OF A LIE ALGEBRA
}

\author{
ALFONS I. OOMS
}

\begin{abstract}
Let $L$ be a finite dimensional Lie algebra over a field $k$ of characteristic zero, $D(L)$ the quotient division ring of $U(L)$. We compare the center $Z(D(L))$ with $Z(D(H))$ where $H$ is an ideal of $L$ of codimension one.
\end{abstract}

Let $k$ be a field of characteristic zero. We first recall the following result due to Bernat [1; 3, p. 134-135].

LEMMA 1. Let $A$ be an associative algebra over $k$ with a unit element, $B$ a subalgebra containing $1, x \in A$ an element symmetric (i.e. $[x, B] \subset B$ ) and transcendental with respect to $B$ such that $A=B[x]$. Hence every element $a \in A$ can be considered as $a$ polynomial in $x$ with coefficients in $B$. We denote by $\operatorname{deg} a$ the degree (in $x$ ) of $a$. We assume further that $A$ and $B$ have quotient division rings $K$ and $L$ (so that $L$ is the division subring of $K$ generated by $B$ ) and that $\Delta$ is a set of derivations of $A$ leaving stable $B$ such that $\operatorname{deg}(E x) \leqslant 1$ for all $E \in \Delta$. We extend the elements of $\Delta$ to derivations of $K$ and denote by $K^{\Delta}$ the set of elements of $K$ which are annihilated by all $E \in \Delta$. Then the following hold:

(1) $x$ is symmetric and transcendental with respect to $L$ and if $a \in A$ then $\operatorname{deg}_{B} a=$ $\operatorname{deg}_{L} a . L \cap A=B$ and the algebra $L[x]$ has a quotient division ring which is $K$.

(2) $K^{\Delta}$ is the quotient division ring of $L[x]^{\Delta}$.

(3) Suppose $K^{\Delta} \neq L^{\Delta}$ (i.e. $L[x]^{\Delta} \not \subset L$ ) and let $z$ be an element of $L[x]^{\Delta}$ of minimal degree $>0$. Then $z$ is transcendental over $L^{\Delta}, L[x]^{\Delta}=L^{\Delta}[z]$ and $K^{\Delta}=L^{\Delta}(z)$.

COROLlaRY. Let us assume in addition to the hypotheses of the lemma that $\operatorname{deg}(E x)=0$ for all $E \in \Delta$. Then $z$ is of the form $c x+d$ with $c \in L^{\Delta}, c \neq 0$, and $d \in L$.

Proof. Choose $u \in L[x]^{\Delta} \backslash L$. We can find $c_{i} \in L$ such that $u=c_{n} x^{n}+$ $c_{n-1} x^{n-1}+\cdots+c_{1} x+c_{0}$ with $c_{n} \neq 0$ and $n \geqslant 1$. Let $E \in \Delta$; then

$$
E u=\left(E c_{n}\right) x^{n}+c_{n} E x^{n}+\left(E c_{n-1}\right) x^{n-1}+c_{n-1} E x^{n-1}+\cdots+E c_{0} .
$$

On the other hand, for each $m \geqslant 1$ we have that

$$
E x^{m}=\sum_{i=1}^{m} x^{m-i}(E x) x^{i-1}=m(E x) x^{m-1}+\text { terms of lower degree. }
$$

Received by the editors June 1, 1982.

1980 Mathematics Subject Classification. Primary 17B35; Secondary 16A08.

Key words and phrases. Finite dimensional Lie algebra, universal enveloping algebra, quotient division ring.

(1)1983 American Mathematical Society $0002-9939 / 82 / 0000-0870 / \$ 01.50$ 
Hence,

(*) $\quad E u=\left(E c_{n}\right) x^{n}+\left(n c_{n} E x+E c_{n-1}\right) x^{n-1}+$ terms of lower degree.

This is a polynomial with coefficients in $L$ since $E c, \in L$ and $E x \in B \subset L$. Now, $u \in L[x]^{\Delta}$ implies that $E u=0$ for all $E \in \Delta$. Taking into account that $x$ is transcendental with respect to $L$, we obtain $E c_{n}=0$ and $n c_{n} E x+E c_{n-1}=0$ so that $E\left(n c_{n} x+c_{n-1}\right)=0$ for all $E \in \Delta$. Consequently, $n c_{n} x+c_{n}, \in L[x]^{د}$ with $c_{n} \in$ $L^{د}, c_{n} \neq 0$. Therefore $\operatorname{deg} z=1$. Finally, it follows easily that $z=c x+d$ for some $c \in L^{\lrcorner}, c \neq 0, d \in L$.

REMARK. If we assume furthermore that $A^{د} \neq B^{د}$ (i.e. $A^{د} \not \subset B$ and a fortiori $K^{\Delta} \neq L^{\Delta}$ ), then a similar argument shows that we can find an element $z=b_{1} x+b_{2}$ $\in A^{\Delta}$ with $b_{1} \in B^{\Delta}, b_{1} \neq 0$, and $b_{2} \in B$ such that $L[x]^{\Delta}=L^{\Delta}[z]$ and $K^{\Delta}=L^{\Delta}(z)$.

Next, let $L$ be a finite dimensional Lie algebra over $k, U(L)$ its universal enveloping algebra and $D(L)$ the quotient division ring of $U(L)$. We denote by $Z(U(L))$ (resp. $Z(D(L)))$ the center of $U(L)$ (resp. $D(L))$. We now recall the following result due to Dixmier [2, p. 339].

LEMMA 2. Let $H$ be an ideal of $L$ of codimension one. If $Z(U(L)) \not \subset U(H)$ then $Z(U(H)) \subset Z(U(L))$ and $Z(U(H))=Z(U(L)) \cap U(H)$.

It follows that we have either $Z(U(L)) \subset Z(U(H))$ or $Z(U(H)) \subset Z(U(L))$.

We are now in a position to prove the following

THEOREM. Let $H$ be an ideal of $L$ of codimension one, $x \in L \backslash H$. Then we have:

1. (a) If $Z(D(L)) \subset Z(D(H))$ then $Z(U(L)) \subset Z(U(H))$.

(b) If $Z(D(H)) \subset Z(D(L))$ then $Z(U(H)) \subset Z(U(L))$.

2. (a) If $Z(U(L)) \subset Z(U(H))$ then $Z(D(L)) \subset Z(D(H))$.

(b) If $Z(U(H)) \underset{\neq}{\subset^{\ddagger}} Z(U(L))$ then $Z(D(H)) \subset_{\neq}^{\mp} Z(D(L))$ and in this case $Z(D(L))$ is a purely transcendental extension of $Z(D(H))^{\neq}$of degree one. Indeed, there exists an element $z=b_{1} x+b_{2} \in Z(U(L))$, transcendental over $Z(D(H))$, with $b_{1} \in Z(U(H))$, $b_{1} \neq 0$, and $b_{2} \in U(H)$ and such that $Z(D(L))$ is generated as a field $b y=$ and $Z(D(H))$. Finally, $Z(D(H))=Z(D(L)) \cap D(H)$.

Proof. 1. (a) $Z(U(L)) \subset Z(D(L)) \subset Z(D(H)) \subset D(H)$. Hence $Z(U(L)) \subset$ $D(H) \cap U(L)=U(H)$. Consequently $Z(U(L)) \subset Z(U(H))$.

(b) $Z(U(H)) \subset Z(D(H)) \subset Z(D(L))$. Therefore $Z(U(H)) \subset Z(D(L)) \cap U(L)$ $=Z(U(L))$.

2. Put $A=U(L), B=U(H)$ and $\Delta=\operatorname{ad} L$. Then $A, B, x$ and $\Delta$ satisfy the conditions of Lemma 1. Indeed, $U(L)$ and $U(H)$ have quotient division rings $D(L)$ and $D(H)$. Since $H$ is an ideal of $L$, every derivation $E \in \operatorname{ad} L$ leaves stable $U(H)$. This holds in particular for ad $x$, which means that $x$ is symmetric with respect to $U(H)$. By the Poincare-Birkhoff-Witt Theorem $x$ is also transcendental over $U(H)$ and $U(L)=U(H)[x]$. Hence each element of $U(L)$ can be written as a polynomial in $x$ with coefficients in $U(H)$ (say on the left). Since $H$ is an ideal of $L$ of codimension one, we have that $[L, L] \subset H$ and $\operatorname{so} \operatorname{deg}(E x)=0$ for all $E \in \Delta=$ ad $L$. We now proceed with the proof. 
(a) Suppose $Z(U(L)) \subsetneq Z(U(H))$. Take $y \in Z(U(H)) \backslash Z(U(L))$ and consider the derivation $E=\operatorname{ad} y$ in $U(L)$. Clearly, $E(U(H))=0$ and $E x=-\operatorname{ad} x(y) \in U(H)$, $E x \neq 0$. Take now $w \in Z(D(L))$. We can choose elements $u, v \in U(L), v \neq 0$, with $w=u v^{-1}$ and such that $\operatorname{deg} v$ is minimal. From $u=w v$ we obtain $E u=(E w) v+$ $w E v=w E v$. Write $v=c_{n} x^{n}+c_{n-1} x^{n-1}+\cdots+c_{0}$ with $c_{i} \in U(H)$ and $c_{n} \neq 0$. Now suppose $E v \neq 0$. This forces $n \geqslant 1$. Then formula (*) gives us

$$
\begin{aligned}
E v & =\left(E c_{n}\right) x^{n}+\left(n c_{n} E x+E c_{n-1}\right) x^{n-1}+\text { terms of lower degree } \\
& =n c_{n}(E x) x^{n-1}+\text { terms of lower degree, }
\end{aligned}
$$

since $E c_{1}=0$ for all $i$. In particular $\operatorname{deg}(E v)=\operatorname{deg} v-1$, which contradicts the minimality of deg $v$ since $w=(E u)(E v)^{-1}$. Therefore $E v=0$, which implies that $n=0$ and so $v=c_{0} \in U(H)$. It follows that $E u=0$ and $u \in U(H)$. Consequently, $w=u v^{-1} \in D(H)$ and thus $w \in Z(D(H))$. We have shown that $Z(D(L)) \subset$ $Z(D(H))$. Moreover $Z(D(L)) \neq Z(D(H))$ since equality would imply $Z(U(L))=$ $Z(U(H))$ by (1).

(b) Suppose $Z(U(H)) \subset Z(U(L))$. Then $Z(U(L)) \not \subset U(H)$ and $Z(U(H))=$ $Z(U(L)) \cap U(H)$. In other words, $U(L)^{\Delta} \not \subset U(H)$ and $Z(U(H))=U(H)^{\Delta}$. Then the corollary and the remark following it assures the existence of an element $z=b_{1} x+b_{2} \in U(L)^{\lrcorner}=Z(U(L))$ with $b_{1} \in U(H)^{\lrcorner}=Z(U(H)), b_{1} \neq 0$, and $b_{2} \in$ $U(H)$, such that $D(H)[x]^{\Delta}=D(H)^{\Delta}[z]$ and $D(L)^{\Delta}=D(H)^{\Delta}(z)$ with $z$ transcendental over $D(H)^{\Delta}$. Clearly $D(L)^{\Delta}=Z(D(L))$, so it remains to show that $D(H)^{\Delta}=$ $Z(D(H))$. For this purpose we take $w \in Z(D(H))$. Then $0=[w, z]=\left[w, b_{1}\right] x+$ $b_{1}[w, x]+\left[w, b_{2}\right]=b_{1}[w, x]$. Hence $[w, x]=0$ which implies that $w \in Z(D(L))$. So we have proved that $Z(D(H)) \subset Z(D(L))$. It follows that

$$
Z(D(H))=Z(D(L)) \cap D(H)=D(L)^{\Delta} \cap D(H)=D(H)^{\Delta} .
$$

Corollary. Let $H$ be an ideal of $L$ of codimension one such that $Z(U(H)) \neq$ $Z(U(L))$. Then we have either $Z(D(H)) \underset{\neq}{\subset} Z(D(L))$ or $Z(D(L)) \underset{\neq}{\subset} Z(D(H))$.

\section{REFERENCES}

1. P. Bernat, Sur le corps enceloppant d' une algèbre de Lie résoluble. Bull. Soc. Math. France. Mèm. No. 7 (1966). MR 37 \#334

2. J. Dixmier, Sur les représentations unitaires des groupes de Lie nilpotents. II. Bull. Soc. Math. France 85 (1957), 325-388. MR 20 \# 1928

3. Algèbres enveloppantes. Cahiers Scientifiques, fasc. 37. Gauthier-Villars, Paris, 1974. MR 58 $16803 a$

Department of Mathematics, Universitair Centrum Limburg, 3610 Diepenbeek. Belgium 